\title{
ORIGINAL ARTICLE \\ Hybrid sterility and evolution in Hawaiian Drosophila: differential gene and allele-specific expression analysis of backcross males
}

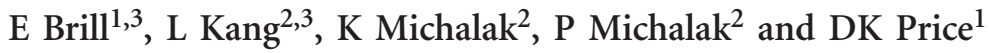 \\ The Hawaiian Drosophila are an iconic example of sequential colonization, adaptive radiation and speciation on islands. \\ Genetic and phenotypic analysis of closely related species pairs that exhibit incomplete reproductive isolation can provide \\ insights into the mechanisms of speciation. Drosophila silvestris from Hawai'i Island and Drosophila planitibia from Maui are \\ two closely related allopatric Hawaiian picture-winged Drosophila that produce sterile $F_{1}$ males but fertile $F_{1}$ females, a pattern \\ consistent with Haldane's rule. Backcrossing $F_{1}$ hybrid females between these two species to parental species gives rise to \\ recombinant males with three distinct sperm phenotypes despite a similar genomic background: motile sperm, no sperm \\ (sterile), and immotile sperm. We found that these three reproductive morphologies of backcross hybrid males produce divergent \\ gene expression profiles in testes, as measured with RNA sequencing. There were a total of 71 genes significantly differentially \\ expressed between backcross males with no sperm compared with those backcross males with motile sperm and immotile \\ sperm, but no significant differential gene expression between backcross males with motile sperm and backcross males with \\ immotile sperm. All of these genes were underexpressed in males with no sperm, including a number of genes with previously \\ known activities in adult testis. An allele-specific expression analysis showed overwhelmingly more cis-divergent than trans- \\ divergent genes, with no significant difference in the ratio of cis- and trans-divergent genes among the sperm phenotypes. \\ Overall, the results indicate that the regulation of gene expression involved in sperm production likely diverged relatively \\ rapidly between these two closely related species.
}

Heredity (2016) 117, 100-108; doi:10.1038/hdy.2016.31; published online 25 May 2016

\section{INTRODUCTION}

Reduced hybrid fitness in the form of hybrid sterility can play an important role in speciation by acting as a post-zygotic isolating barrier (Coyne and Orr, 2004). Haldane (1922) first documented that in crosses between species sterility is more likely to occur in hybrid individuals of the heterogametic sex. Since then, this observation has been shown to occur in almost all animals; especially in Drosophila spp., and is called 'Haldane's rule' (Coyne and Orr, 2004). In Drosophila species, hybrid male sterility (HMS) has been shown to function as an evolutionarily early limiting factor to introgression between species and consequently maintain species integrity (Noor and Feder, 2006). Hybrid incompatibility and sterility are thought to occur when epistatic interactions of alleles from different species are dysfunctional (Johnson, 2000), creating incompatible developmental pathways, ecological detriments or altered (and therefore, unsuccessful) mating behavior (Coyne and Orr, 2004). Genetic models have been developed that indicate that this type of fitness reduction may require at least two genetic changes - one from each species-but can be much more complex and be the result of multiple gene interactions (Johnson, 2000; Coyne and Orr, 2004). Multiple studies of HMS in the well-studied allopatric species pair Drosophila mauritiana and Drosophila simulans showed interactions of at least three genes or more (Johnson, 2000), and the identification of over 100 genes contributing to HMS on the X chromosome (Wu et al., 1996), as well as many genes on the autosomes (Tao et al., 2003; Araripe et al., 2010; Dickman and Moehring, 2013).

In addition to extensive $\mathrm{X}$ and autosomal HMS loci studies, gene expression studies in Drosophila have shown a number of spermatogenesis genes differentially expressed between hybrids and parental species (Landry et al., 2007a). Theoretical models suggest that the effects of accumulation of regulatory incompatibilities in the architecture of transcriptional networks can be a part of hybrid incompatibilities directly influencing the process of speciation (Porter and Johnson, 2002; Johnson and Porter, 2007). Techniques such as microarrays have been used to characterize gene expression, and have uncovered several genes that are deregulated in hybrids between species, especially in D. mauritiana and D. simulans (Michalak and Noor, 2003; Moehring et al., 2007). D. mauritiana/D. simulans sterile male $\mathrm{F}_{1}$ hybrids are more likely to succumb to downregulation of genes associated specifically with male reproduction, implying a

${ }^{1}$ Tropical Conservation Biology and Environmental Science Graduate Program, University of Hawai'i at Hilo, Hilo, HI, USA and ${ }^{2}$ Biocomplexity Institute, Virginia Tech, Blacksburg, VA, USA

Correspondence: Dr P Michalak, Biocomplexity Institute, Virginia Tech, Blacksburg, VA 24061, USA or Dr DK Price, Tropical Conservation Biology and Environmental Science Graduate Program, University of Hawai'i at Hilo, Hilo, HI 96720, USA.

E-mail: pawel@vbi.vt.edu or donaldp@hawaii.edu

${ }^{3}$ These authors contributed equally to this work.

Received 12 January 2016; revised 18 February 2016; accepted 24 February 2016; published online 25 May 2016 
possible genetic cause to their sterility (Michalak and Noor, 2003, 2004). Closely related species pairs can provide further insights into the genetic mechanisms of speciation because of incomplete reproductive isolation in the form of sterile $\mathrm{F}_{1}$ males but fertile $\mathrm{F}_{1}$ females that can be backcrossed to parental species. Analyzing the differences in gene expression among backcrossed (BC) individuals will provide insights into the variation in expression of testis-specific genes and also to potential candidate genes that lead to reproductive isolation by comparing transcriptomes between sterile and fertile BC siblings. Such a comparison between backcross hybrids having similar genomic backgrounds but distinct fertility phenotypes provides a higher resolution of the association between genes and phenotypes than that between $\mathrm{F}_{1}$ hybrids and parental species, as every subsequent generation of backcrossing on average halves the amount of HMS-unrelated heterozygosity (Michalak and Noor, 2004).

HMS is generally observed to occur earlier in the divergence of species (that is, more closely related species) than hybrid inviability, suggesting stronger selection on sterility-causative genes (Orr et al., 2004). DNA sequence divergence and expression levels of sex-related genes in many studies support the idea that genes involved in male fertility diverge faster between species than other types of genes (Orr et al., 2004). Hybrid sterility-causative genes, such as Odysseus site homeobox (OdsH) in Drosophila (Ting et al., 1998), Meisetz (Prdm9) in mice (Oliver et al., 2009) and AEP2/OLI1 in yeast (Lee et al., 2008), are often characterized by rapid sequence evolution and distinct expression patterns. Candidate genes for speciation, therefore, include genes responsible for spermatogenesis and sperm motility and other genes that cause reproductive incompatibilities in hybrids.

HMS is an important post-zygotic reproductive isolating mechanism in many eukaryotic organisms, but only recently has genome-wide gene expression analysis been used to investigate the full suite of genes involved in the expression of this complex trait (Gomes and Civetta, 2015). Here we analyze the differential gene expression and allelespecific expression (ASE) of testis-level fertility in BC males between two closely related endemic Hawaiian Drosophila species in the picture-wing clade under planitibia group and IV $\beta$ subgroup, Drosophila planitibia and Drosophila silvestris (Spieth, 1986). D. silvestris is endemic to Hawai'i Island and D. planitibia is endemic to the island of Maui, and diverged $~ 0.7$ Mya (O'Grady et al., 2011; Magnacca and Price, 2015). Both species are bark breeders, whereby females oviposit eggs on and larvae develop in the decaying bark of the endemic Hawaiian flowering plant, Clermontia spp. ('oha wai) (Magnacca et al., 2008). The subgroup is known for selecting leks in more open spaces as opposed to other subgroups that prefer a hidden location. Current phylogenetic analyses show that $D$. planitibia shares a close ancestor with both $D$. silvestris and $D$. heteroneura as the latter two species were established on Hawai'i Island (Magnacca and Price, 2015).

Previous studies have shown that successful mating can occur between the two species, creating sterile $\mathrm{F}_{1}$ hybrid males, but fertile $\mathrm{F}_{1}$ hybrid females (Craddock, 1974). Fertile $F_{1}$ females can be used to create a backcross generation whereby males exhibit different sperm phenotypes. Using the RNA sequencing platform and a de novo transcriptome assembly, we identified 71 differentially expressed (DE) genes across three $\mathrm{BC}$ phenotypes, showing a clear underexpression of key functional genes in $\mathrm{BC}$ individuals that lack sperm compared with those individuals who possess large numbers of motile sperm. These results demonstrate a directional gene expression change correlated with HMS, providing important insight into the mechanisms of reproductive isolation.

\section{MATERIALS AND METHODS}

\section{Drosophila stocks}

The D. planitibia and D. silvestris populations used in this study as parental population were initiated from individuals recently captured from wild populations: $\sim 20 \mathrm{D}$. planitibia individuals captured in Waikamoi Preserve, east Maui (GPS coordinates 20.811286, - 156.241901), in December 2012 and $\sim 20$ D. silvestris individuals collected in South Kona Forest Reserve, Kukuiopae unit (GPS coordinates 19.297281, - 155.811710), Hawai'i Island in June 2013. All parental, $\mathrm{F}_{1}$ and $\mathrm{BC}$ populations were maintained in a controlledenvironment room maintained at a constant $18{ }^{\circ} \mathrm{C}, 70 \%$ relative humidity with a 12:12 light/dark cycle. This is a standard environment for rearing Hawaiian Drosophila (Uy et al., 2015) with a generation time of 3-4 months. Adults were housed in 4-1 glass jars with a damp fine sand floor and containing 3 to 5 vials of adult food. The $25 \times 95 \mathrm{~mm}$ adult food vials contained a yeastless Wheeler-Clayton medium and a small tissue moistened with a tea made with the leaves of Clermontia spp. on which females oviposited as described by Carson (1987). Adult food vials in which females deposited eggs on tissue were replaced weekly and placed in larvae-rearing trays. Larvae food, a yeastcornmeal-molasses medium, was provisioned to each vial with active larvae several times per week. At 4 weeks, maturing larvae were transferred to emergence jars containing moistened large-grain sand for burial pupation. Emerging adult flies were aspirated from emergence jars twice a week and separated into male or female jars to ensure flies were virgins for future experiments.

Adult D. silvestris and D. planitibia used to produce $\mathrm{F}_{1}$ and $\mathrm{BC}$ offspring were $\sim 14-21$ day post eclosion when the flies reach reproductive maturity and placed in mating vials $(28.5 \times 95 \mathrm{~mm})$ that contained adult food and a tissue moistened with Clermontia spp. tea. Each cross was labeled, dated and numbered to ensure identification. Adults were transferred to new mating vials each week to ensure ideal mating and egg-laying conditions. The old mating vials were placed in larvae trays labeled with type of cross, date and identification number and fed larvae food. As larvae reached the third instar, the larval vials were placed in 2-1 pupation/emergence jars, and emerging adult male and female flies were removed weekly from the pupation/emergence jars, before becoming sexually mature, and segregated into jars by each species or hybrid and sex. Hybrids and BCs were attempted during parental generations $\mathrm{F}_{4}-\mathrm{F}_{7}$. The production of $\mathrm{BC}$ individuals was accomplished by mating $\mathrm{F}_{1}$ hybrid females (from the $D$. silvestris females $\times D$. planitibia males cross) to emerged $D$. silvestris males of the parental species after flies reached sexual maturity (Figure 1). Because of the difficult nature of obtaining hybrids and backcrosses, all $\mathrm{BC}$ males used in this study were full siblings from one successful $\mathrm{F}_{1}$ hybrid female/D. silvestris parental male pair.

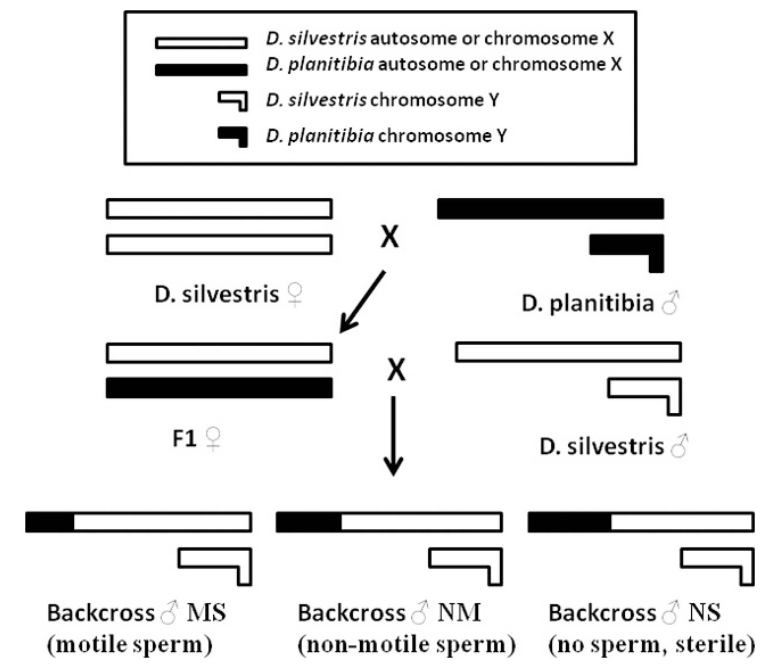

Figure 1 Schematic diagram of backcrossing of $F_{1}$ D. silvestris $\times$ $D$. planitibia female to $D$. silvestris with three distinct sperm phenotypes among male offspring. 


\section{Testes dissection and RNA collection}

D. planitibia, D. silvestris, $\mathrm{F}_{1}$ hybrid males and $\mathrm{BC}$ adult male flies that had reached sexual maturity (4 weeks post eclosion) were dissected at room temperature under similar housing and stabilization conditions using sterilized Dumont No. 5 fine forceps (Dumont, Montignez, Switzerland), tungsten needles and a $100 \times 20 \mathrm{~mm}$ glass dissection dish that were sprayed with RNaseZAP (Sigma-Aldrich, St Louis, MO, USA) before each dissection to remove RNase contamination. Males were housed in their own individual glass vials with food and dissected after a 24 -h stabilization period in the laboratory. Testes were dissected under a compound light microscope. One testis and its accessory glands were bifurcated and placed into a RNase-free $2 \mathrm{ml}$ roundbottomed tube (Eppendorf, Hamburg, Germany) filled with RNALater (Life Technologies, Carlsbad, CA, USA). The other testis was prepared for a live testis squash by placing the tissue on a $20 \times 20 \mathrm{~mm}$ cover slip with $10 \mu \mathrm{l}$ drop of testis buffer and covered with a clean glass slide. The prepared slide was inverted and placed under a compound light microscope at $\times 10$ and $\times 40$ magnification for observation.

$\mathrm{BC}$ males were categorized into three phenotype groups based on testis and sperm observations and cross-referenced with Craddock's classifications (Craddock, 1974): males that exhibited complete absence of mature sperm (BC-NS) and were thus sterile, males that had sperm that was nonmotile and often clumped inside the testes (BC-NM) and males that had motile sperm (BC-MS). Because of the low numbers of mature BC males in the laboratory, we did not have extra $\mathrm{BC}$ males to conduct fertility experiments to determine the ability of BC-MS and BC-NM males to father progeny.

\section{Sample preparation and sequencing}

Twenty-four testis samples were prepared for RNA extraction: 3 D. planitibia, 3 D. silvestris, 2 D. silvestris $\times D$. planitibia $\mathrm{F}_{1}$ hybrids, 7 BC-MS, 4 BC-NM and 5 BC-NS. RNA extraction and sequencing were conducted at the Virginia Bioinformatics Institute, Virginia Tech (Blacksburg, VA, USA) and University of Hawaici at Hilo (Hilo, HI, USA). Total RNA was extracted using Trizol Reagent (Life Technologies) following the manufacturer's instructions. Using TruSeq RNA sample preparation kit (Illumina, FC-122-1001/1002), mRNA from $1 \mu \mathrm{g}$ of total RNA with RNA integrity number $\geqslant 8.0$ was converted into a library of template molecules suitable for subsequent cluster generation and sequencing with Illumina HiSeq 2500 (Illumina, San Diego, CA, USA). The libraries generated were validated using Agilent 2100 Bioanalyzer (Agilent, Santa Clara, CA, USA) and quantitated using Quant-iT dsDNA HS Kit (Invitrogen, Waltham, MA, USA) and quantitative PCR. Individually indexed complementary DNA libraries were pooled, clustered onto a flow cell using Illumina's TruSeq SR Cluster Kit v3 (GD-401-3001) and sequenced for 101 cycles using two TruSeq SBS Kit HS (FC-401-1002) on HiSeq 2500.

\section{de novo transcriptome assembly}

Adapter sequences were removed from the raw sequencing reads. Duplicated and low-quality reads were discarded using FastqMcf (Aronesty, 2013) with default parameters. To exclude possible contamination, all reads were aligned to a bacterial database downloaded from National Center for Biotechnology Information (NCBI), and only unmapped reads were used to assemble the transcriptome. The processed reads from all samples were merged together and assembled with Trinity (Grabherr et al., 2011) (with parameter -trimmomatic). TransDecoder (Broad Institute, Cambridge, MA, USA) was used to identify candidate coding regions within assembled transcripts, and transcripts with open reading frame lengths $<300$ (100 amino acids) were filtered out. The final transcriptome assembly was used as a reference for gene annotation and expression calculation.

\section{Gene annotation}

Transcripts/genes were mapped to NR database (nonredundant protein database from NCBI) using BLAST (v. 2.2.28, NCBI). Alignments with threshold $e$-values $>1 \mathrm{e}-20$ or identity $<50 \%$ were discarded. The $e$-values determine significant matches to the database by describing the number of hits by chance. The top hit from Drosophila species was used to annotate the query genes (giving priority to Drosophila melanogaster, if present), and genes without Drosophila hits were discarded to further exclude possible contamination.

\section{Gene expression}

The clean reads were mapped to the reference assembly using Bowtie (Langmead et al., 2009) (v. 1.0.0) with parameters set to ' -125 -I 1 -X 1000 -a $-\mathrm{m}$ 200' (seed length 25 , the minimum and maximum insert sizes for paired-end alignment of 1 and 1000, respectively, and report alignments <200). RSEM (Li and Dewey, 2011) was used to calculate the gene expression with default parameters. The differential expression of genes was calculated using the DEseq (Anders and Huber, 2010) package in R software (http://www.r-project.org/), with Benjamini-Hochberg adjusted $P$-values $<0.05$ considered to be significant.

\section{Allele-specific expression}

RNA sequencing reads were mapped to the reference transcriptome using BWA (v. 0.7.6; Li and Durbin, 2010) with default parameters. Genotypes were identified by UnifiedGenotyper from GATK package (McKenna et al., 2010) with default parameters except setting heterozygosity to 0.01 and indel heterozygosity to 0.00125 . Genotypes with genotyping quality $<40$ or mapping quality $<30$, or covered depth $<10$ were discarded. Only homozygous speciesspecific loci from parental D. planitibia and D. silvestris samples were chosen as ASE candidate sites. To reduce the bias of reference preference during the mapping process, a masked reference was generated by changing the reference to a third genotype different from the homozygous genotypes in D. planitibia and D. silvestris in all ASE candidate positions. Genotypes for other samples were generated as above, using the masked sequence as reference. Loci of heterozygous genotypes in $\mathrm{F}_{1}$ and $\mathrm{BC}$ samples with a minimal genotyping score of 40, mapping score of 30 and coverage depth of 10 were tested with a binomial test, and candidates with Benjamini-Hochberg adjusted $P$-values of $<0.05$ were kept. Simulated reads around the candidate ASE sites were generated with half of the reads carrying the $D$. silvestris genotype and the other half carrying the D. planitibia genotype. Simulated reads that mapped against the masked reference and the simulated genotypes generated with binomial test $P$-value of $<0.05$ were excluded from the ASE candidate pool as false positives. As the analysis was confined to heterozygous loci, we were unable to detect extreme cases of ASE in which only one allele was expressed. This bias against extreme ASE leads to an underestimate of the total ASE levels but at the same time makes our analysis more conservative.

Using a general framework of cis/trans divergence with respect to allelic ratios (Wittkopp et al., 2004; Landry et al., 2005), we formulated cis-score $\left(S_{c i s}\right)$ and trans-score $\left(S_{\text {trans }}\right)$ as follows:

$$
\begin{aligned}
& S_{c i s}=1-\frac{1}{4} \cdot \mid(|p-f|+|p-b|) \\
& S_{\text {trans }}=1-\left(\frac{1}{4} \cdot|f-b|+\frac{1}{4} \cdot(|f|+|b|)\right)
\end{aligned}
$$

where $p, f$, and $b$ are allelic ratios for parental, $\mathrm{F}_{1}$ and $\mathrm{BC}$ samples, respectively. For each gene, $P=\left(E_{\text {pla }}-E_{\text {sil }}\right) /\left(E_{\text {pla }}+E_{\text {sil }}\right)$ was calculated as the ratio based on the gene expressions of D. planitibia $\left(E_{\text {pla }}\right)$ and D. silvestris $\left(E_{\text {sil }}\right) ; f=\left(C_{\text {pla }}-C_{\text {sil }}\right) /$ $\left(C_{p l a}+C_{s i l}\right)$ was calculated as a ratio based on the number of reads corresponding to the D. planitibia allele $\left(C_{\text {pla }}\right)$ and the number of reads corresponding to the D. silvestris $\left(C_{\text {sil }}\right)$ allele in $\mathrm{F}_{1}$ samples; $b$ was calculated in a similar way as $f$ but in BC samples. The gene was called either cis- or trans-divergent in each BC group when one score was larger than the other. If the difference of the two scores was $<0.05$, the gene was classified as cis/trans synergy. This formalization is consistent with the idea that cis divergence pattern is characterized by equal parental $(p)$ and hybrid $(f)$ ratios, whereas trans divergence pattern is characterized by the $f$ ratio (but not necessarily $p$ ratio) approaching zero. We expanded the framework to include heterozygous $\mathrm{BC}$ hybrids, assuming that sequence divergence exerts the same ASE effects in $\mathrm{F}_{1}$ and backcross hybrids, and hence $f$ and $b$ should be convergent. In addition to each phenotypic BC group classified separately, all BC individuals (combined BC) were analyzed as one group for a combined $\mathrm{BC}$ cis/trans divergence measure. A subset of 800 genes containing informative single-nucleotide polymorphism between D. planitibia and D. silvestris were used for this analysis.

\section{Gene Ontology (GO) enrichment}

All functional enrichment analyses were carried out on the DAVID (Database for Annotation, Visualization and Integrated Discovery) site (v. 6.7) (http:// 
david.abcc.ncifcrf.gov/). The list of DE genes was uploaded to the DAVID website that cross-referenced the gene IDs among many databases to provide gene ontology annotations as well as categorizing the genes into broader functional groups.

\section{Interaction network}

Interaction network for cis- and trans-divergent genes was generated using GeneMania (Mostafavi et al., 2008), whereas DroID (Yu et al., 2008) data were incorporated as an additional data source.

\section{RESULTS}

Testes and sperm morphologies

Parental species males $(N=42 D$. silvestris, $N=44$ D. planitibia) consistently exhibited fully formed testes with dense, coiled sperm that was motile after the testis squash (Figures $2 \mathrm{a}$ and $\mathrm{b}$ ). $\mathrm{F}_{1}$ hybrid males $(N=23)$ were sterile, containing no sperm inside an intact testis (Figure 2c). Full-sib BC males showed a range of sperm morphologies (Figures 2d-f) consistent with the results reported in Craddock (1974). BC-MS males $(N=19)$ exhibited a large number $(>80 \%)$ of highly motile sperm inside an intact testis (Figure 2d); BC-NM males $(N=15)$ exhibited nonmotile sperm in intact testes (Figure 2e); and BC-NS males $(N=15)$ exhibited a completely empty testis where no sperm was present similar to $\mathrm{F}_{1}$ hybrid males (Figure $2 \mathrm{f}$ ). Of the $16 \mathrm{BC}$ individuals dissected in this study, the distribution of the three sperm phenotype categories were as follows: 7 were BC-MS (motile sperm), 4 were BC-NM (nonmotile sperm present) and 5 were BC-NS (completely sterile, absence of sperm).

\section{Transcriptome assembly and annotation}

After quality control for raw sequencing reads, an average of $2.84 \mathrm{~Gb}$ clean data per sample was generated. Clean reads from all samples were used in the de novo transcriptome assembly. Trinity output yielded 102981 genes (170 887 transcripts) with an average contig/ transcript length of 554 base pairs (bp). To assure enrichment of mRNAs, contigs were post-processed by filtering out open reading frames $<300 \mathrm{bp}$. A remaining total of 24320 genes $(55038$ transcripts) with average length of $991 \mathrm{bp}$ and total length of $54.5 \mathrm{Mb}$ were used for mapping. Out of the 24320 genes, 21307 (87.6\%) mapped to the NR database under our criteria, with 16761 (68.9\%) of them with hits to Drosophila. Gene IDs were assigned according to the hits, with the majority coming from the wellannotated D. melanogaster (13 655) and D. grimshawi (2933), the only Hawaiian Drosophila species sequenced to date.

\section{Analysis of differential gene expression and candidate genes}

Gene expression was compared among BC groups. There were 65 genes significantly DE between BC-MS and BC-NS and 33 between $\mathrm{BC}-\mathrm{NM}$ and $\mathrm{BC}-\mathrm{NS}$, but no significant differential gene expression was found between BC-MS and BC-NM (Figure 3, Supplementary Figure 1 and Supplementary Table 1). Interestingly, all significantly DE genes found between BC-MS and BC-NS, as well as BC-NM and BCNS, were downregulated in BC-NS. For comparison, 41 genes showed higher expression in $\mathrm{BC}-\mathrm{MS}$ and 30 genes had higher expression in $\mathrm{BC}-\mathrm{NM}$ for the comparison between BC-MS and BC-NM, although differential expression was not statistically significant for any of these genes (Supplementary Table 1 and Supplementary Figure 1).

Among the $33 \mathrm{DE}$ genes between BC-NM and BC-NS, 27 were shared in DE genes between BC-MS and BC-NS groups. The most significant shared DE gene was CG31467, known to be expressed at moderate levels in D. melanogaster adult testis according to FlyAtlas (http://flyatlas.org/). No significant GO enrichment was found for DE genes, presumably because of the relatively low level of functional annotation. Cellular components, biological processes and molecular functions for each gene hit were recorded if the data were available in Flybase (Table 1). Nevertheless, we have analyzed GO term enrichments for the top $5 \%$ misexpressed genes in the three comparisons between BC groups (Supplementary Table 2). The analysis showed high similarities between the comparisons, especially between BC-MS/ $\mathrm{BC}-\mathrm{NS}$ and $\mathrm{BC}-\mathrm{NM} / \mathrm{BC}-\mathrm{NS}$, in which GO terms related to microtubule cytoskeleton were among the most overrepresented.
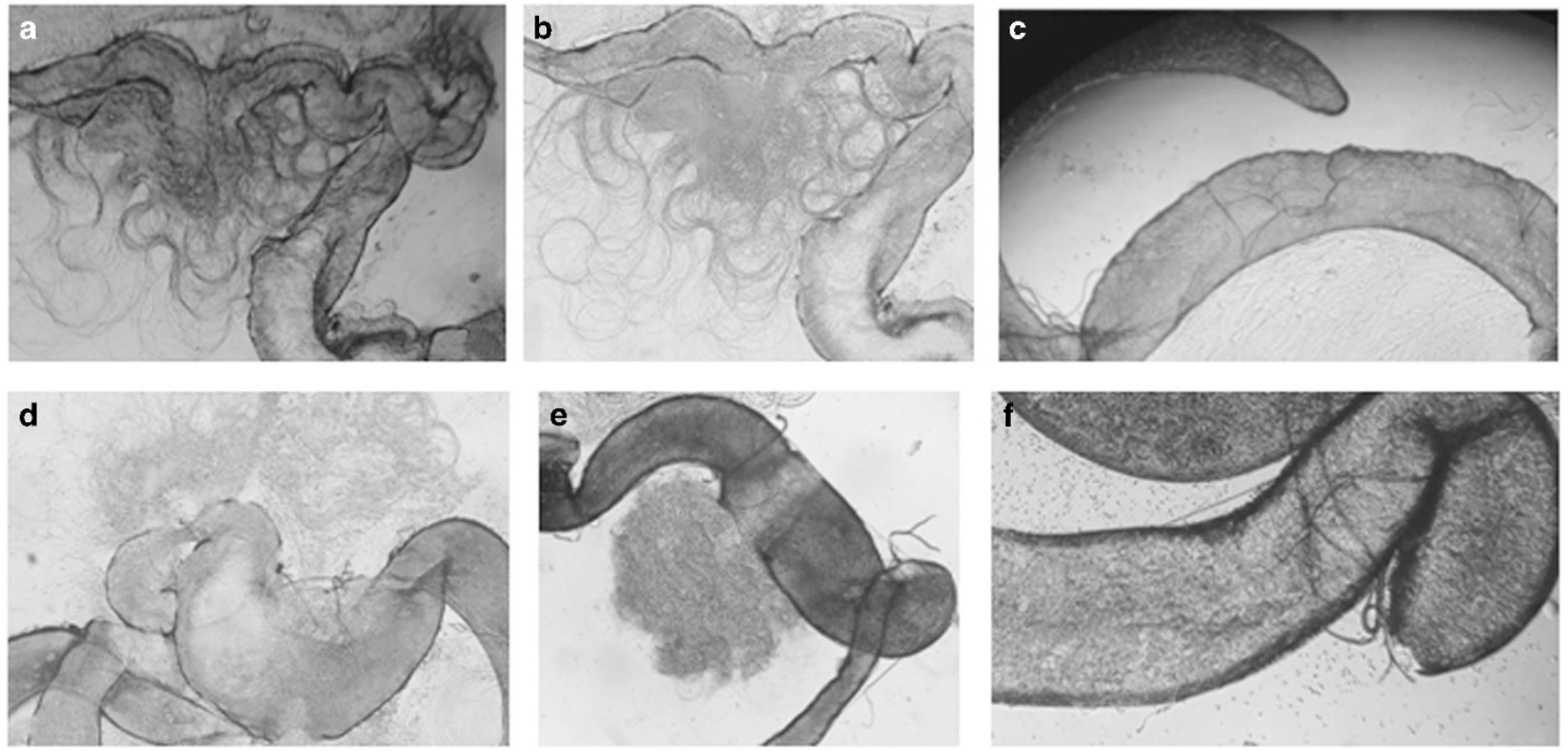

Figure 2 Photographs of representative testis phenotypes post testis squash exhibited by (a) D. silvestris, (b) D. planitibia, (c) $F_{1}$ hybrid, (d) BC-MS (motile sperm), (e) BC-NM (sperm present but non-motile) and (f) BC-NS (no sperm present). All dissections and photographs were taken under a light compound microscope at either $\times 10$ or $\times 40$ magnification. 
a

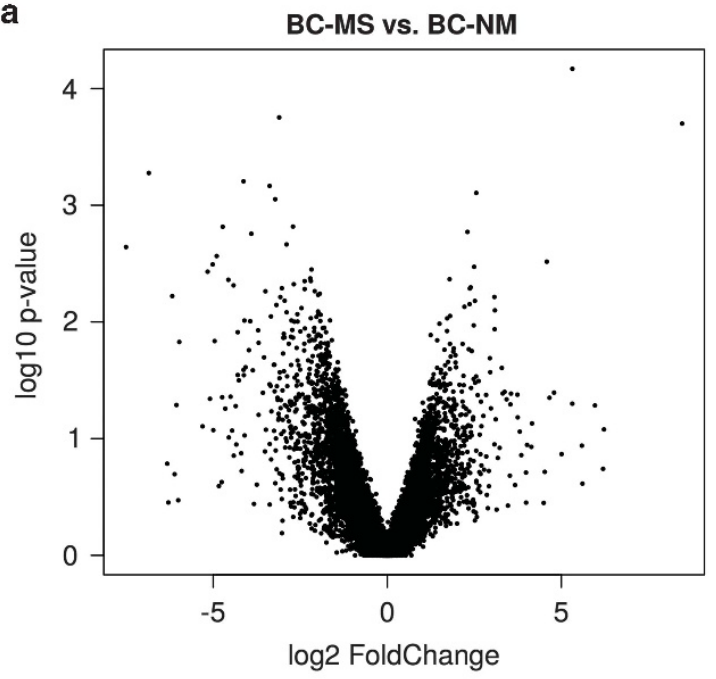

C

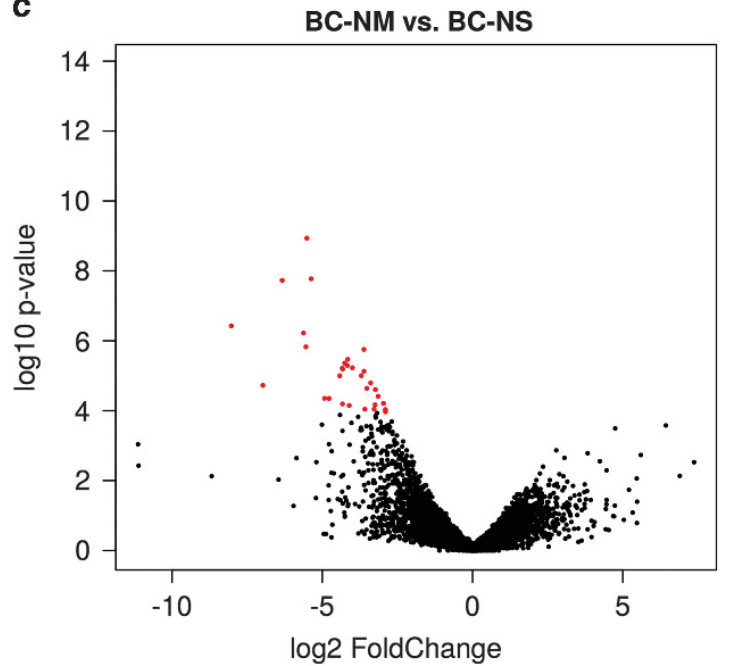

b

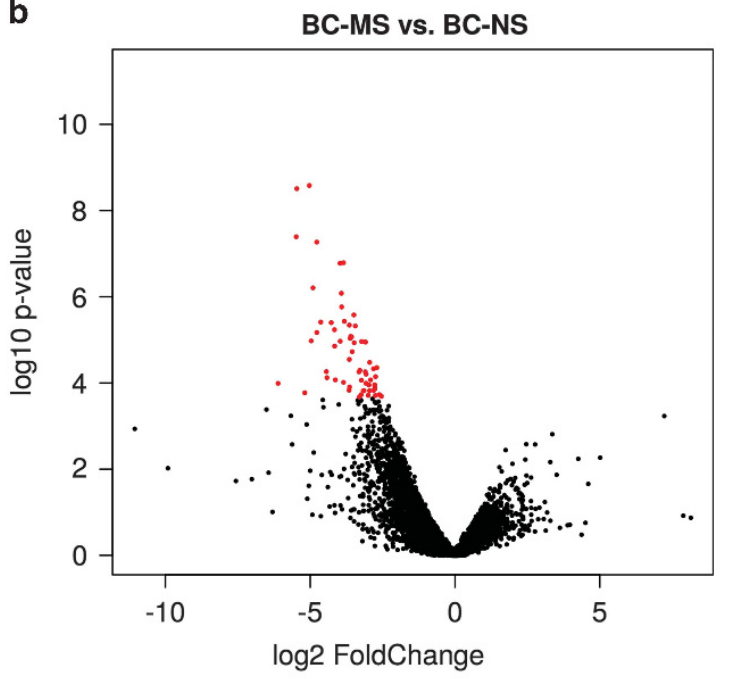

Figure 3 Volcano plot for differential expression analysis among the testes of backcross male groups. The x-axis is the log2 fold change of the differences in gene expression (a): BC-MS > BC-NM, (b) BC-MS > BC-NS and (c) BC-NM > BC-NS. The y-axis is the negative of the log10 false discovery rate (FDR) values of the comparison between the two $\mathrm{BC}$ male groups, with red dots indicating significant $\mathrm{DE}$ genes.

Of the 65 DE genes between BC-MS and BC-NS, 4 genes have known spermatogenesis-related function(s) in $D$. melanogaster (Table 1). Of the $33 \mathrm{DE}$ genes between BC-NM and BC-NS, at least three genes have spermatogenesis-related function: $k l-5$, pendulin and CG15161 (Table 1). Other important functions represented by the remaining DE genes include microtubule activities (LP11180p, $k l-2$, Dgri GH10382, gb:AAN71272.1), phosphatase binding (CG31467, Dgri IGH13925), oxidation-reduction processes (DgrilGH19779, Dgri IGH22053, Dgri $G H 17535)$ and hydrolase activities (Dgri GH11455, $P p V)$. Of the 71 DEs found among BC groups, all of them also showed differential expression between parental species and BC-NS, and 42 of them showed differential expression between parental species and both BC-MS and BC-NM.

\section{ASE analysis}

There were more cis-divergent than trans-divergent genes with no significant difference in the ratio of cis- and trans-regulated genes among three BC male groups $\left(\chi^{2}=1.29\right.$, d.f. $=2, P=0.52$, Table 2$)$. BC-MS had 335 cis-regulated, 97 trans-regulated and 169 synergistic genes; BC-NM had 283 cis-regulated, 67 trans-regulated and 125 synergistic genes; BC-NS had 333 cis-regulated, 90 trans-regulated and 143 synergistic genes; and the combined BC groups had 333 cisregulated, 108 trans-regulated and 180 synergistic genes (Table 2). Out of the 71 significantly misexpressed genes (Table 1 and Supplementary Table 1), only four had polymorphism (species-specific singlenucleotide polymorphisms in transcribed sequences and heterozygosity in backcrosses) permitting computations of cis/trans scores and cis/ trans divergence classification. Two out of the four genes were cis/trans synergistic (Octbeta3R and CG5196) and two were cis/regulated (DgrilGH10450 and DgrilGH13801). To test for a possible contribution of compensatory cis/trans evolution (manifested as misregulation of cis/trans synergistic genes in hybrids; Landry et al., 2005) to backcross hybrid sterility, we estimated Spearman's rank correlations between cis/trans score differences (being low for cis/trans synergy) and expression fold changes between the BC groups. The correlation was positive but not significant $(r=0.04, P=0.34)$, inconsistent with the scenario that compensatory cis/trans evolution significantly contributed to differences between the BC groups. In addition, an interactome network of cis- and trans-divergent genes was generated (Supplementary Figure 2). 
Table 1 Differentially expressed genes among the testes of BC-MS, BC-NM and BC-NS and their known biological processes and molecular functions

\begin{tabular}{|c|c|c|c|c|}
\hline Gene IDa & $F / S^{b}$ & $N M / S^{b}$ & Biological processes & Molecular function \\
\hline 1,2CG31467 & $\mathrm{S}$ & $\mathrm{S}$ & Negative regulation of phosphatase activity & Phosphatase binding \\
\hline $1,2 \mathrm{kl}-5$ & $\mathrm{~S}$ & NS & Microtubule-based movement & ATPase activity, coupled; motor activity \\
\hline${ }^{2}$ pendulin & $\mathrm{S}$ & NS & $\begin{array}{l}\text { Centrosome duplication; lymph gland development; sperm } \\
\text { individualization }\end{array}$ & Protein transmembrane transporter activity \\
\hline 1,2 Octbeta3R & S & NS & G protein-coupled receptor signaling pathway & G protein-coupled amine receptor activity \\
\hline $1,2 L P 11180 p$ & S & NS & Microtubule-based movement & Motor activity; ATPase activity, coupled \\
\hline${ }^{2}$ DgrilGH10450 & $\mathrm{S}$ & NS & Metabolic processes; transmembrane transport & Catalytic activity \\
\hline 1,2 pen & S & S & Apposition of dorsal and ventral imaginal disc-derived wing surfaces & RNA binding \\
\hline${ }^{2}$ DgrilGH13925 & $\mathrm{S}$ & $\mathrm{S}$ & Negative regulation of phosphatase activity & Phosphatase binding \\
\hline 1,2DgrilGH19779 & S & $\mathrm{S}$ & Oxidation-reduction process & Oxidoreductase activity \\
\hline 1,2DgrilGH20554 & S & S & rRNA transcription & Endoribunuclease activity \\
\hline${ }^{1,2}$ DgrilGH11455 & $\mathrm{S}$ & $\mathrm{S}$ & Metabolic process & Hydrolase activity \\
\hline${ }^{2}$ CG5196 & S & S & Golgi organization; protein palmitoylation & $\begin{array}{l}\text { Protein-cysteine S-palmitoyltransferase activity; zinc ion } \\
\text { binding }\end{array}$ \\
\hline${ }^{1,2}$ CG5718 & S & $\mathrm{S}$ & Electron transport chain; tricarboxylic acid cycle & Succinate dehydrogenase (ubiquinone) activity \\
\hline 1,2lobo (lost boy) & S & NS & Sperm motility; sperm storage & NA \\
\hline 1,2DgrilGH10382 & S & NS & Microtubule nucleation & NA \\
\hline${ }^{2} P p V$ & $\mathrm{~S}$ & S & Mitotic cell cycle; protein dephosphorylation & Hydrolase activity \\
\hline${ }^{2}$ Prosbeta7 & S & S & $\begin{array}{l}\text { Cell proliferation; cellular response to DNA Damage stimulus; mitotic } \\
\text { spindle }\end{array}$ & Endopeptidase activity \\
\hline${ }^{2}$ DgrilGH11042 & S & NS & Protein phosphorylation & ATP binding; protein kinase activity \\
\hline${ }^{2}$ CG15161 & S & $\mathrm{S}$ & Cilium assembly; intraciliary transport & NA \\
\hline $1,2 \mathrm{kl}-2$ & S & NS & Microtubule-based movement & ATP binding; ATPase activity, coupled; motor activity \\
\hline${ }^{2} Z n T 33 D$ & S & S & Cellular zinc ion homeostasis & Zinc ion transmembrane transporter activity \\
\hline${ }^{2}$ DgrilGH23359 & $\mathrm{S}$ & $\mathrm{S}$ & Protein glycosylation & Fucosyltransferase activity \\
\hline${ }^{1,2}$ CG9173 & $\mathrm{S}$ & NS & Regulation of cell cycle & NA \\
\hline${ }^{1,2}$ DgrilGH22053 & $\mathrm{S}$ & NS & NA & Cytochrome- $c$ oxidase activity \\
\hline${ }^{1,2}$ DgrilGH10111 & S & NS & Lipid metabolic process & Phosphoric diester hydrolase activity; starch binding \\
\hline${ }^{2}$ DgrilGH15386 & S & NS & NA & Lysozyme activity \\
\hline${ }^{2} T p c 2$ & S & NS & Transmembrane transport & Transmembrane transporter activity \\
\hline${ }^{1,2}$ DgrilGH17535 & $\mathrm{S}$ & NS & Oxidoreductase activity & Oxidation-reduction process \\
\hline${ }^{2}$ Vha16-1 & S & NS & dsRNA transport; imagine disc-derived wing morphogenesis & Hydrogen ion transmembrane transporter activity \\
\hline${ }^{2}$ DgrilGH25085 & $\mathrm{S}$ & NS & Metabolic process & Catalytic activity \\
\hline${ }^{2} b r v 3$ & NS & $\mathrm{S}$ & Calcium ion transport & Calcium channel activity; calcium ion binding \\
\hline${ }^{2}$ DgrilGH16238 & NS & S & Protein glycosylation & Fucosyltransferase activity \\
\hline${ }^{2} g b: A A N 71272.1$ & $\mathrm{~S}$ & S & Microtubule-based movement & Motor activity; ATPase activity, coupled \\
\hline 1,2 unc 80 & NS & $\mathrm{S}$ & Locomotor rhythm & Cation channel activity \\
\hline
\end{tabular}

Abbreviations: BC, backcrosses; BC-MS; backcrosses with motile sperm; BC-NM, backcrosses with sperm present but nonmotile; BC-NS, backcrosses with no sperm present; dsRNA; doublestranded RNA; NA, not available; NS, nonsignificant differential expression; S, significant differential expression.

Only genes with known functions are included in this table.

asuperscript 1 indicates differential expression between parental species and both BC-MS and BC-NM; superscript 2 indicates differential expression between parental species and BC-NS.

${ }^{\mathrm{b}} \mathrm{F} / \mathrm{S}$ and NM/S indicate comparison between BC-MS and BC-NS, and between BC-NM and BC-NS, respectively.

Table 2 Total number of cis-divergent, trans-divergent and cis/trans synergistic genes expressed in the testes of each $\mathrm{BC}$ group and all BC groups combined

\begin{tabular}{lccc}
\hline BC group & cis & trans & cis/trans \\
\hline BC-MS & 335 & 97 & 169 \\
BC-NM & 283 & 67 & 125 \\
BC-NS & 333 & 90 & 143 \\
All BC & 333 & 108 & 180
\end{tabular}

Abbreviations: BC, backcrosses; BC-MS; backcrosses with motile sperm; BC-NM, backcrosses with sperm present but nonmotile; BC-NS, backcrosses with no sperm present.

\section{DISCUSSION}

\section{Sperm morphologies}

Our analysis of sperm production and motility in this study is consistent with the study of Craddock (1974) showing similar proportions of the three fertility phenotypic groups in the $\mathrm{BC}$ males. The results show that the underlying physiological processes in the $\mathrm{BC}$ males of these two species results in three distinct phenotypic classes with one group lacking sperm in the testis (BC-NS), a second group with nonmotile sperm (BC-NM) and a third group with testis filled with motile sperm (BC-MS). The $\mathrm{F}_{1}$ hybrid males are similar to the sterile $\mathrm{BC}$ males (BC-NS) and both parental species are similar to the fertile $\mathrm{BC}$ males (BC-MS). These results also suggest that HMS may be caused by two processes with one disrupting sperm production and the other disrupting sperm motility.

\section{Differential gene expression}

Our RNA sequencing analyses on BC adult male testes also showed highly significant differential gene expression between two of the three phenotypic groups of $\mathrm{BC}$ males. There were $65 \mathrm{DE}$ genes observed between BC-MS and BC-NS and 33 DE genes between BC-NM and 
BC-NS. Three potential candidate genes of interest are identified as D. melanogaster genes lost boys, male fertility factor $k l-2$ and male fertility factor kl-5. The lost boys is a gene involved in ciliar motility and encodes a conserved flagellar protein CG34110 that is localized along fly sperm flagella and is highly expressed in ciliated respiratory epithelia and sperm (Yang et al., 2011). Phenotypic analysis in D. melanogaster showed that lost boys specifically affected sperm movement into the female storage receptacle (Yang et al., 2011). Therefore, it is a gene that is involved directly in sperm motility and sperm storage.

Male fertility factor $k l-2$ and male fertility factor $k l-5$ are two genes that reside in the long arm of the $\mathrm{Y}$ chromosome in D. melanogaster, and are two of seven fertility factors identified in D. melanogaster (Carvalho et al., 2000). Deletion studies showed that the lack of kl-5 results in the loss of the outer arm of the sperm tail axoneme; sperm in males lacking $k l-2$ and $k l-5$ were missing important heavy chain proteins, and therefore the individuals produced immotile sperm (Carvalho et al., 2000). kl-5 is known to code for an axonemal $\beta$-dynein heavy chain expressed in the testis; these heavy chains are known to be responsible for the motility of flagella and cilia (Carvalho et al., 2000). We note that all of the BC males in this study have the same D. silvestris $\mathrm{Y}$ chromosome. If $k l-2$ and $k l-5$ genes are on the $\mathrm{Y}$ chromosome of $D$. silvestris and D. planitibia, it would suggest that in order for there to be DE between the backcross males, given that all have the same $\mathrm{Y}$ chromosome, $k l-2$ and $k l-5$ genes would have to interact with genes or regulatory factors located elsewhere in the genome on either an autosome or X chromosome. The construction of chromosomal maps for these species would be valuable in determining whether these genes are located on the $\mathrm{Y}$ chromosome.

Other functionally annotated genes of interest in this set include CG31467 and Octbeta3R. CG31467 was the top DE gene and plays a role in phosphatase binding and is expressed moderately in the adult testis of D. melanogaster (FlyBase). Protein phosphatases have been known to modulate sperm motility in mammals (Fardilha et al., 2011), and a gene encoding acylphosphatase (Acyp) has been found to be associated with HMS in $\mathrm{F}_{1}$ and backcross hybrids between $D$. simulans and D. mauritiana (Michalak and Ma, 2008; Michalak and Noor, 2004). Octbeta3R is homologous to $\beta$-adrenergic receptors in vertebrates that play multifunctional roles in insects, and is an octopamine (Farooqui, 2012). In D. melanogaster, octopamines have been detected in pathways relating to different behaviors such as olfactory learning and memory, aggression, locomotion and grooming and conditional courtship (Farooqui, 2012). Octbeta3R specifically has been known to partially restore ovulation and fecundity in sterile females.

$\mathrm{DE}$ genes between BC-MS and BC-NS and between BC-NM and $\mathrm{BC}$-NS were all downregulated in the sterile phenotype that lacked sperm (BC-NS) compared with the fertile phenotype (BC-MS) and the nonmotile sperm phenotype (BC-NM). This result suggests that the sterile phenotype does not express testis function genes compared with the purported fertile phenotype, as expected. It should be noted that many of the downregulated genes in BC-NS do not necessarily need to be responsible for, or even associated with, HMS. Indeed, many of these genes are also downregulated in $\mathrm{BC}-\mathrm{MS}$ and $\mathrm{BC}-\mathrm{NM}$ relative to the parental species, suggesting that this misexpression may be a more general feature of backcross hybrids regardless of their fertility phenotype. On the other hand, it would also be premature to claim that such genes cannot be related to fertility for this reason, as we cannot rule out a possibility that even fertile backcross males are subfertile relative to parental species. Another complication in this and other studies of gene expression in HMS is due to tissue structural alterations, including gonadal atrophies and sperm deficiencies, leading to spurious expression changes among genes with tissuespecific activity, difficult (if possible at all) to distinguish from true gene silencing. As BC-NS males lack sperm, genes with sperm-specific expression will necessarily be underrepresented in the analysis. To minimize the effect of sperm absence on the expression profile, we excluded genes with zero-level expression.

Interestingly, we did not find significant differential expression between BC-MS and BC-NM. Both BC-MS and BC-NM contain sperm, but BC-MS contains many motile sperm, and BC-NM contains less dense, nonmotile sperm. Although such sperm motility-related genes as lost boys, male fertility factor kl-2 and male fertility factor kl-5 were significantly underexpressed in BC-NS, their expression alterations were subtler in $\mathrm{BC}-\mathrm{NM}$. Indeed, there were a number of genes that exhibited differential expression between the BC-MS and BC-NM phenotypic groups that did not reach statistical significance with the sample sizes in this study (Figure 3). For both BC-MS/BC-NS and $\mathrm{BC}-\mathrm{NM} / \mathrm{BC}-\mathrm{NS}$, the top DE gene was CG31467 that is moderately expressed in the testis. Alternatively, BC-NM phenotype could be conveyed through regulatory changes at post-transcriptional, translational or post-translational levels, including protein-protein interactions. Lastly, we did not capture interfamily variation, as all BC males used in this study were offspring from a single $\mathrm{F}_{1}$ hybrid female/D. silvestris parental male pair, and this has its advantages (genetically related brothers with distinct phenotypes) and also disadvantages (difficulty with extrapolations to the population levels).

\section{Allele-specific expression}

The interaction of cis- and trans-regulatory factors during transcription can affect gene expression (reviewed in Bell et al., 2013), and both factors are subject to mutational changes that may result in transcriptional alterations. Sensitivity of gene expression to mutations increases with both increasing trans-mutational target size and the presence of a TATA box (Landry et al., 2007b). These regulatory networks are primarily composed of regulatory and structural genes (Wittkopp et al., 2004). We found overwhelmingly more cis-divergent genes than trans-divergent genes in all $\mathrm{BC}$ groups. This result is consistent with those from other Drosophila, including closely related Drosophila $p$. pseudoobscura and D. p. bogotana (Gomes and Civetta, 2015), as well as more distantly related $D$. melanogaster and $D$. simulans (Wittkopp et al., 2004, 2008). A review of regulatory experiments by Bell et al. (2013) concludes that generally cis-regulatory changes account for more divergent expression between more genetically divergent parents (for example, interspecific) than trans-effects that account for a higher proportion of variation in gene expression between less divergent parents (for example, intraspecific).

Wittkopp et al. (2004) models of regulatory divergence suggest structural genes tend to be more cis-regulatory than trans regulatory because of their proximity to terminal nodes of the network where expression of genes is not regulated within the network. In contrast, some of the cis genes of interest had regulatory functions. However, we did not observe an excess of DNA-binding gene activities (or other significant GO term enrichments) among trans-divergent genes (Supplementary Tables 3). The most abundant groups of genes among cis-divergent genes were functionally related to cytoskeleton (12 genes) and reproductive cellular processes (7 genes). Unlike Landry et al. (2005), we did not observe increased misexpression of cis $\times$ trans synergistic genes that is likely related to the fact that comparisons between backcross hybrids display less extensive misexpressions than comparisons between $F_{1}$ hybrids and parental species (Michalak and Noor, 2004), whereas cis $\times$ trans synergy does not disproportionally contribute to backcross sterility. 


\section{Evolutionary implications of regulatory divergence}

The combination of cis- and trans-regulatory elements in these BC groups allows further insight into the gene expression within and between groups, and the potential genetic architecture contributing to their divergence. Gene regulation has been shown to be heritable (Pavey et al., 2010; Yang et al., 2014) and an important part of divergence among species and variation within populations, but cis- and trans-acting elements differ in their evolutionary influence (Meiklejohn et al., 2014). For example, if cis elements are stronger than trans elements, then changes in a species pair may have evolved one gene at a time, instead of through a broad sweep as would be inferred by a trans-dominant regulation that affects many genes (Wittkopp et al., 2004). Understanding regulatory networks can lead to further insight into the potential associations among gene expression, adaptive genetic divergence and reproductive isolation, as this link is not conclusive in many experiments and needs further testing (Pavey et al., 2010). The combination of molecular techniques and fitness assays will provide a more robust analysis of the relationship between isolation and divergence relative to gene expression (Pavey et al., 2010). These studies could also be used as a proxy for comparing and contrasting the ecological divergence of other pairs of closely related Hawaiian Drosophila species, such as the sympatric, strongly reproductively isolated D. heteroneura and D. silvestris. Potential differences in cis- and trans-acting factors may be important in providing a more robust analysis and unraveling the evolutionary histories among species.

Overall, the HMS between the closely related D. silvestris and $D$. planitibia species highlights the relatively rapid divergence of gene regulation for testis development and sperm production in Hawaiian Drosophila. The physiological and developmental processes that are involved in testis formation and sperm production appear to have diverged to such an extent that the two systems have become incompatible within $\sim 0.7$ million years. The evolution of these species on separate islands also suggests that selection operating separately on male gamete production within each species is an important contributor to divergence between these two species. Furthermore, the potential for interactions between genes on the $\mathrm{Y}$ chromosome with genes or genetic factors located either on the $\mathrm{X}$ chromosome or autosomes is consistent with the proposition that this sterility occurs, at least in part, through epistatic interactions of alleles from the two species, which leads to incompatible systems (Johnson, 2000). As gonad formation begins in the larval stage and is maintained into the adult stage (Williamson and Lehmann, 1996), it will be important to examine gene expression patterns throughout the developmental process to determine where the compatibility of the genomes breaks down in the formation of testes and the production of sperm. Further research is also necessary to examine the potential for a smaller number of genes to create the initial incompatible developmental pathways that then lead to other changes in developmental and gene expression patterns to create hybrid male sterility (Coyne and Orr, 2004; Johnson, 2000). The comparison of gamete formation between more species in a phylogenetic context could shed light into how gene expression patterns and the gamete physiological and developmental systems evolve over time within and between species (Porter and Johnson, 2002; Johnson and Porter, 2007).

\section{DATA ARCHIVING}

Data were deposited at NCBI SRA under accession number SRP068366.

\section{CONFLICT OF INTEREST}

The authors declare no conflict of interest.

\section{ACKNOWLEDGEMENTS}

We thank Renee Corpuz for assisting in raising the Hawaiian picture wing Drosophila and Anne Veillet at the University of Hawaii at Hilo Core Genomics Facility for assisting with the dissections and RNA extractions. We thank Betsy Gagné, Cynthia King and Lyman Perry of the Hawaii Department of Land and Natural Resources, Division of Forestry and Wildlife, for permission to collect Hawaiian Drosophila and Mark Vaught of the East Maui Irrigation Co. for permission to collect in the Waikamoi Forests in East Maui. Three anonymous reviewers provided valuable suggestions for improving the manuscript. This research is based upon work supported by the National Science Foundation CREST Center award EHR-0833211 to DKP.

Anders S, Huber W (2010). Differential expression analysis for sequence count data. Genome Biol 11: R106.

Araripe LO, Montenegro H, Lemos B, Hartl DL (2010). Fine-scale genetic mapping of a hybrid sterility factor between Drosophila simulans and D. mauritiana: the varied and elusive functions of "speciation genes". BMC Evol Biol 10: 385.

Aronesty E (2013). Comparison of sequencing utility programs. The Open Bioinform $J$ 7: $1-8$.

Bell GD, Kane NC, Rieseberg LH, Adams KL (2013). RNA-seq analysis of allele-specific expression, hybrid effects, and regulatory divergence in hybrids compared with their parents from natural populations. Genome Biol Evol 5: 1309-1323.

Carson HL (1987). High fitness of heterokaryotypic individuals segregating naturally within a long-standing laboratory population of Drosophila silvestris. Genetics 116: 415-422.

Carvalho AB, Lazzaro BP, Clark AG (2000). Y chromosomal fertility factors kl-2 and kl-3 of Drosophila melanogaster encode dynein heavy chain polypeptides. Proc Natl Acad Sci USA 97: 13239-13244.

Coyne JA, Orr HA (2004). Speciation vol. 37, Sinauer Associates: Sunderland, MA, USA.

Craddock EM. Reproductive relationships between homosequential species of Hawaiian Drosophila. Evolution 28: 593-606.

Dickman CT, Moehring AJ (2013). A novel approach identifying hybrid sterility QTL on the autosomes of Drosophila simulans and D. mauritiana. PLoS One 8: e73325.

Fardilha M, Esteves SL, Korrodi-Gregorio L, Pelech S, da Cruz ESOA, da Cruz ESE (2011). Protein phosphatase 1 complexes modulate sperm motility and present novel targets for male infertility. Mol Hum Reprod 17: 466-477.

Farooqui T (2012). Review of octopamine in insect nervous systems. Open Access Insect Physiol 4: 1-17.

Gomes S, Civetta A (2015). Hybrid male sterility and genome-wide misexpression of male reproductive proteases. Sci Rep 5: 11976.

Grabherr MG, Haas BJ, Yassour M, Levin JZ, Thompson DA, Amit I et al. (2011). Full-length transcriptome assembly from RNA-Seq data without a reference genome. Nat Biotechnol 29: 644-652.

Haldane JB (1922). Sex ratio and unisexual sterility in hybrid animals. J Genet 12: $101-109$.

Johnson N (2000). Gene interactions and the origin of species. In: Wade MJ (ed), Epistasis and the Evolutionary Process. Oxford University Press: New York. pp 197-212.

Johnson NA, Porter AH (2007). Evolution of branched regulatory genetic pathways: directional selection on pleiotropic loci accelerates developmental system drift. Genetica 129: 57-70.

Landry CR, Hartl DL, Ranz JM (2007a). Genome clashes in hybrids: insights from gene expression. Heredity (Edinb) 99: 483-493.

Landry CR, Lemos B, Rifkin SA, Dickinson WJ, Hartl DL (2007b). Genetic properties influencing the evolvability of gene expression. Science 317: 118-121.

Landry CR, Wittkopp PJ, Taubes CH, Ranz JM, Clark AG, Hartl DL (2005). Compensatory cis-trans evolution and the dysregulation of gene expression in interspecific hybrids of Drosophila. Genetics 171: 1813-1822.

Langmead B, Trapnell C, Pop M, Salzberg SL (2009). Ultrafast and memory-efficient alignment of short DNA sequences to the human genome. Genome Biol 10: R25.

Lee HY, Chou JY, Cheong L, Chang NH, Yang SY, Leu JY (2008). Incompatibility of nuclear and mitochondrial genomes causes hybrid sterility between two yeast species. Cel/ 135 . 1065-1073.

Li B, Dewey CN (2011). RSEM: accurate transcript quantification from RNA-Seq data with or without a reference genome. BMC Bioinformatics 12: 323.

Li H, Durbin R (2010). Fast and accurate long-read alignment with Burrows-Wheeler transform. Bioinformatics 26: 589-595.

Magnacca KN, Foote D, O'Grady PM (2008). A review of the endemic Hawaiian Drosophilidae and their host plants. Zootaxa 1728: 1-58.

Magnacca KN, Price DK (2015). Rapid adaptive radiation and host plant conservation in the Hawaiian picture wing Drosophila (Diptera: Drosophilidae). Mol Phylogenet Evol 92 226-242.

McKenna A, Hanna M, Banks E, Sivachenko A, Cibulskis K, Kernytsky A et al. (2010). The Genome Analysis Toolkit: a MapReduce framework for analyzing next-generation DNA sequencing data. Genome Res 20: 1297-1303. 
Meiklejohn CD, Coolon JD, Hartl DL, Wittkopp PJ (2014). The roles of cis- and transregulation in the evolution of regulatory incompatibilities and sexually dimorphic gene expression. Genome Res 24: 84-95.

Michalak P, Ma D (2008). The acylphosphatase (Acyp) alleles associate with male hybrid sterility in Drosophila. Gene 416: 61-65.

Michalak P, Noor MA (2003). Genome-wide patterns of expression in Drosophila pure species and hybrid males. Mol Biol Evol 20: 1070-1076.

Michalak P, Noor MA (2004). Association of misexpression with sterility in hybrids of Drosophila simulans and D. mauritiana. J Mol Evol 59: 277-282.

Moehring AJ, Teeter KC, Noor MA (2007). Genome-wide patterns of expression in Drosophila pure species and hybrid males. II. Examination of multiple-species hybridizations, platforms, and life cycle stages. Mol Biol Evol 24: 137-145.

Mostafavi S, Ray D, Warde-Farley D, Grouios C, Morris Q (2008). GeneMANIA: a real-time multiple association network integration algorithm for predicting gene function. Genome Biol 9 (Suppl 1): S4.

Noor MA, Feder JL (2006). Speciation genetics: evolving approaches. Nat Rev Genet 7: 851-861.

O'Grady PM, Lapoint RT, Bonacum J, Lasola J, Owen E, Wu Y et al. (2011). Phylogenetic and ecological relationships of the Hawaiian Drosophila inferred by mitochondrial DNA analysis. Mol Phylogenet Evol 58: 244-256.

Oliver PL, Goodstadt L, Bayes JJ, Birtle Z, Roach KC, Phadnis N et al. (2009). Accelerated evolution of the Prdm9 speciation gene across diverse metazoan taxa. PLoS Genet 5: e1000753.

Orr HA, Masly JP, Presgraves DC (2004). Speciation genes. Curr Opin Genet Dev 14: 675-679.

Pavey SA, Collin H, Nosil P, Rogers SM (2010). The role of gene expression in ecological speciation. Ann NY Acad Sci 1206: 110-129.

Porter AH, Johnson NA (2002). Speciation despite gene flow when developmental pathways evolve. Evolution 56: 2103-2111.
Spieth HT (1986). Behavioral characteristics of Hawaiian Drosophila. Proc Hawaiian Entomol Soc 26: 101-108.

Tao Y, Zeng ZB, Li J, Hartl DL, Laurie CC (2003). Genetic dissection of hybrid incompatibilities between Drosophila simulans and D. mauritiana. II. Mapping hybrid male sterility loci on the third chromosome. Genetics 164: 1399-1418.

Ting CT, Tsaur SC, Wu ML, Wu Cl (1998). A rapidly evolving homeobox at the site of a hybrid sterility gene. Science 282: 1501-1504.

Uy KL, LeDuc R, Ganote C, Price DK (2015). Physiological effects of heat stress on Hawaiian picture-wing Drosophila: genome-wide expression patterns and stressrelated traits. Conserv Physiol 3: 1-14.

Williamson A, Lehmann R (1996). Germ cell development in Drosophila. Annu Rev Cell Dev Biol 12: 365-391.

Wittkopp PJ, Haerum BK, Clark AG (2004). Evolutionary changes in cis and trans gene regulation. Nature 430: 85-88.

Wittkopp PJ, Haerum BK, Clark AG (2008). Regulatory changes underlying expression differences within and between Drosophila species. Nat Genet 40: 346-350.

Wu Cl, Johnson NA, Palopoli MF (1996). Haldane's rule and its legacy: Why are there so many sterile males? Trends Ecol Evol 11: 281-284.

Yang S, Liu Y, Jiang N, Chen J, Leach L, Luo Z et al. (2014). Genome-wide eQTLs and heritability for gene expression traits in unrelated individuals. BMC Genomics 15: 13.

Yang Y, Cochran DA, Gargano MD, King I, Samhat NK, Burger BP et al. (2011). Regulation of flagellar motility by the conserved flagellar protein CG34110/Ccdc135/FAP50. Mol Biol Cell 22: 976-987.

Yu J, Pacifico S, Liu G, Finley RL Jr (2008). DrolD: the Drosophila Interactions Database, a comprehensive resource for annotated gene and protein interactions. BMC Genomics 9: 461 .

Supplementary Information accompanies this paper on Heredity website (http://www.nature.com/hdy) 